\title{
Clostridium difficile Colitis
}

\author{
Judith L. Trudel, M.D. ${ }^{1}$
}

Clostridium difficile enterocolitis is endemic in most modern hospitals. The spectrum of clinical presentation varies from the asymptomatic carrier state to fulminant colitis with toxic megacolon and perforation. Highly toxigenic and lethal strains of C. difficile have emerged worldwide. Medical treatment consists of discontinuing the precipitating antibiotic, supportive measures and bowel rest, and antibiotic treatment with metronidazole or vancomycin. Surgical treatment may be necessary in cases of fulminant disease. Subtotal colectomy with end ileostomy is the operation of choice.

KEYWORDS: Clostridium difficile, pseudomembranous enterocolitis, antibioticassociated colitis

Objectives: On completion of this article, the reader should be able to: (1) recognize the wide spectrum of clinical presentations encountered in Clostridium difficile-associated disease and the emergence of new lethal strains worldwide; (2) understand the pathophysiology of Clostridium difficile colitis; and (3) recognize the indications for surgical management of Clostridium difficile colitis.

Clostridium difficile is a gram-positive sporeforming anaerobic microorganism. Clostridium difficileassociated disease (CDAD) is usually secondary to exposure to antibiotics. Antibiotics suppress the normal enteric flora and allow $C$. difficile to overgrow and start producing its toxins. ${ }^{1,2}$ Clostridium difficile enterocolitis, also called antibiotic-induced colitis or pseudomembranous colitis (PMC), is the most frequent nosocomial enteric disease encountered by surgeons. ${ }^{3} \mathrm{CDAD}$ is endemic in most modern hospitals because of the widespread use of prophylactic and therapeutic antibiotherapy. The recent worldwide emergence of highly toxigenic and lethal strains of $C$. difficile ${ }^{4,5}$ emphasizes the need for prevention and aggressive management of this condition.

The spectrum of clinical presentation of CDAD varies from the asymptomatic carrier state to fulminant colitis with or without toxic megacolon or colonic perforation. ${ }^{6}$ Approximately $1 \%$ to $3 \%$ of the healthy adult population and up to $65 \%$ of infants ${ }^{8}$ who have never been exposed to antibiotics are asymptomatic carriers. Asymptomatic carriers may transmit the disease by the fecal-oral route or by spores, which can remain dormant on inanimate objects for weeks to months. Few asymptomatic adult carriers excrete the cytotoxins; in contrast, $55 \%$ of asymptomatic infant carriers excrete toxins but are resistant to the clinical disease during their first year of life because of the immaturity of their enterocyte toxin-receptor sites. ${ }^{7}$

CDAD may arise as self-limited antibioticassociated diarrhea with or without visible pseudomembranes or as classic pseudomembranous disease. Severe cases may progress to fulminant colitis with toxic megacolon or perforation. Resistant disease and relapsing disease (5\% to $30 \%$ of cases) are seen increasingly frequently. ${ }^{9}$

All antibiotics, including metronidazole and vancomycin, ${ }^{10}$ which are used for treatment, have been implicated in the development of PMC; penicillins, ${ }^{7}$ cephalosporins, ${ }^{7}$ clindamycin, ${ }^{7}$ and quinolones ${ }^{11-13}$ are

\footnotetext{
${ }^{1}$ Division of Colon and Rectal Surgery, Department of Surgery, University of Minnesota, St. Paul, Minnesota.

Address for correspondence and reprint requests: Judith L. Trudel, M.D., Division of Colon and Rectal Surgery, Department of Surgery, University of Minnesota, 393 Dunlap Ave. North, \#500, St. Paul, MN 55104. E-mail: jtrudel@colonrectal.org.
}

Miscellaneous Colitides; Guest Editor, Judith L. Trudel, M.D.

Clin Colon Rectal Surg 2007;20:13-17. Copyright (C) 2007 by Thieme Medical Publishers, Inc., 333 Seventh Avenue, New York, NY 10001, USA. Tel: +1(212) 584-4662.

DOI 10.1055/s-2007-970195. ISSN 1531-0043. 
associated with the highest incidence of disease. Even a single dose of antibiotics may lead to CDAD. Immunosuppressed patients such as elderly patients, transplant patients, or cancer patients undergoing antineoplastic therapy are particularly susceptible to CDAD and need not be exposed to antibiotics to develop clinical disease.

\section{PATHOPHYSIOLOGY}

C. difficile produces at least two identifiable toxins, $A$ and B. ${ }^{6}$ Toxin $A$ is an enterotoxin and cytotoxin; it initiates an inflammatory reaction that increases colonic permeability to toxin B. Toxin B, which is the toxin measured routinely in the cytotoxin assay, is also a potent cytotoxin. Both toxins combine to produce profuse watery diarrhea. C. difficile also produces collagenase, which increases mucosal breakdown and facilitates bacterial translocation. Toxins and bacteria may thus enter the portal circulation and undergo phagocytosis by hepatic macrophages. This results in release of chemomediators, such as interleukin 1 , interleukin 6 , and tumor necrosis factor, manifested by the septic systemic response observed in many patients.

Some new, more pathogenic strains of $C$. difficile have been identified worldwide since 2001. These highly toxigenic strains of $C$. difficile secrete larger quantities of toxins $\mathrm{A}$ and $\mathrm{B}$ and also secrete an additional clostridial toxin called "binary toxin." 4 The mechanism of action of this toxin is unclear, but its contribution to the increased toxicity and lethality of the disease is indisputable. 4,9,14,15 "Modern CDAD" is characterized by an increase in the number of cases and severity of the disease, a decrease in response to treatment, an increase in the frequency of recurrences, and the emergence of community-acquired strains of the disease. ${ }^{9}$

Although most of the toxin effect is directed against colonocytes, the jejunum and ileum are also susceptible to disease. C. difficile enteritis may be seen in patients who have a permanent ileostomy after colectomy ${ }^{16}$ or patients who have an ileoanal reservoir after total proctocolectomy. The clinical presentation is identical to that of $C$. difficile colitis.

\section{CLINICAL PRESENTATION AND DIAGNOSIS}

C. difficile colitis is characterized by profuse watery diarrhea (90\% to $95 \%)$, crampy abdominal pain $(80 \%$ to $90 \%$ ), and fever and leukocytosis (80\% each). $3,6,7,17$ Bloody diarrhea is distinctly uncommon; its occurrence should trigger a search for inflammatory bowel disease or other colitides. Nonspecific malaise is often present. Recent antibiotic usage is reported in the vast majority of the cases; immunosuppressed patients may not have been exposed to antibiotics yet develop the disease. The onset of diarrhea typically occurs within 7 days of initiation and within 2 to 21 days of cessation of antibiotherapy. Unless the patient presents with fulminant disease and an acute abdomen, physical findings are few and nonspecific.

The diagnosis is made by confirming the presence of toxin B in the stool by enzyme-linked immunosorbent assay (ELISA) testing. Stool cultures are not routinely required for diagnosis. Toxin assay allows rapid detection ( 2 to 4 hours) of the toxin. The sensitivity and specificity of ELISA testing are $70 \%$ to $95 \%$ and $99 \%$ to $100 \%$, respectively. Stool cultures for C. difficile are more sensitive but are more time consuming (usually a few days) and expensive, and their usefulness is somewhat limited by the $3 \%$ incidence of healthy asymptomatic carriers.

The endoscopic appearance of $C$. difficile colitis is pathognomonic. The colonic mucosa is peppered with multiple whitish plaques of varying sizes, measuring a few millimeters to 1 to $2 \mathrm{~cm}$. The plaques are usually raised and adherent and may be confluent when severe disease is present. When visible, the intervening colonic mucosa may look edematous, granular, hyperemic, or completely normal. Unfortunately, pseudomembranes are present in only $\sim 50 \%$ of patients who are toxin assay positive. Furthermore, the distribution of pseudomembranes is patchy and segmental, with as many as $70 \%$ of patients presenting with rectal sparing and as many as $10 \%$ of patients presenting with typical pseudomembranes above the reach of the flexible sigmoidoscope. Proctoscopy is therefore unreliable for diagnosis. At a minimum, flexible sigmoidoscopy should be performed; in suspicious cases where flexible sigmoidoscopy is normal, colonoscopy should be considered strongly. The endoscopic appearance is so typical that biopsies are unnecessary to confirm the diagnosis. If performed, biopsies of the plaques show epithelial debris, polymorphonuclear infiltrate, chronic inflammation, and fibrin deposition.

A computed tomography (CT) scan is often performed routinely in patients with abdominal pain of uncertain etiology. CT findings of PMC are nonspecific and include diffuse pancolonic wall thickening, mucosal edema, thumbprinting, doughnut-like appearance of the ascending and descending colon, and colonic dilatation. The small bowel is usually spared and appears normal radiologically. Ileus may be present. Patients with toxic megacolon and perforation may also demonstrate free fluid or free air, or both.

\section{MEDICAL TREATMENT OF PSEUDOMEMBRANOUS COLITIS}

The two goals of treatment are (1) to improve the patient's clinical condition and (2) to prevent the spread of CDAD to other patients. Most of the time, these two goals are achievable simultaneously, yet there is debate over possible unnecessary treatment in selected patients. 
The first line of treatment for all patients is to discontinue the offending antibiotic. This may be the only treatment needed in mild forms of PMC. Symptom resolution is seen within 2 to 3 days of antibiotic cessation. Patients whose diarrhea resolved simply on stopping antibiotics may continue to shed $C$. difficile in their feces for several weeks and therefore remain infectious. The importance of general hygiene precautions (hand washing, use of disposable medical devices, etc.) cannot be overemphasized. Contamination of environmental surfaces by $C$. difficile spores resistant to cleaning and disinfection is a well-documented phenomenon. ${ }^{9}$ This is particularly important in hospitals where crowded wards, suboptimal toilet-to-bed ratios, and highly selected populations of at-risk patients are encountered.

Use of antidiarrheals (e.g., loperamide, Lomotil ${ }^{\mathbb{R}}$ ) is contraindicated in all cases of pseudomembranous enterocolitis as this may exacerbate toxin-mediated disease and precipitate toxic megacolon. ${ }^{3,7,18}$ Intravenous hydration and bowel rest should be considered if clinically indicated.

In more severe cases, treatment directed against C. difficile is recommended. Several antibiotics, including vancomycin, metronidazole, bacitracin, and teicoplanin (not readily available in United States), are effective in providing symptomatic and bacteriologic cure. ${ }^{19}$ The most effective route of administration is oral. In the United States, treatment with either metronidazole $(500 \mathrm{mg}$ orally three times a day for 10 to 14 days) or vancomycin (125 mg orally four times a day for 7 days) is recommended. ${ }^{3,6,7,17,20}$ Metronidazole should be the initial agent of choice; it is cheaper and more versatile than vancomycin because intravenous metronidazole is effective against C. difficile and parenteral vancomycin is not. In addition, the emergence of vancomycin-resistant enterococcus strains supports limiting use of vancomycin to patients in whom initial treatment with metronidazole has failed. Vancomycin may also be delivered by enemas or ileostomy lavage if indicated $(500 \mathrm{mg}$ in $1 \mathrm{~L}$ of saline three times daily). For patients intolerant of or allergic to metronidazole and vancomycin, oral bacitracin $(20,000$ to 25,000 units four times daily for 7 to 14 days) may be used.

Anion-exchange resins (e.g., cholestyramine, colestipol) have been used successfully for patients in whom prior antibiotic therapy failed; they are not recommended for initial treatment as their effectiveness is inferior to that of antibiotics. Resins cannot be used in conjunction with vancomycin as they bind the drug as well as the toxin.

Restoration of normal fecal flora has been proposed as a potential approach to prevent and treat $\mathrm{CDAD}$. One recent systematic review of probiotic therapy of $\mathrm{CDAD}$ with various bacterial preparations concluded that there is insufficient evidence to recommend routine use, ${ }^{21}$ whereas another meta-analysis found significant reduction in CDAD with selected probiotics. ${ }^{22}$ Restoration of normal fecal flora with fecal enemas $^{23-25}$ or nasogastric stool infusion ${ }^{26}$ has been described, mostly for resistant relapsing cases, but these modalities have (understandably) met with resistance from patients and are seldom used.

Ninety-five percent of patients treated with antibiotics directed against $C$. diffcile respond to initial therapy. Resistance of $C$. difficile to antibiotics has never been documented, yet the relapse rate varies from $5 \%$ to $30 \% .{ }^{27}$ Relapse is due either to persistence of $C$. difficile in its spore form or to reinfection. The importance of hygiene, infection control measures, and modern physical infrastructures in today's hospitals is paramount. Risk factors for recurrent disease include advanced age, female sex, renal disease, and continued use of antibiotics. Relapses are treated with a repeated course of metronidazole or vancomycin; the response rate to a second course is $92 \%$. A small minority of patients go on to develop chronic relapsing disease and may need prolonged tapering therapy (up to 5 weeks) with vancomycin. $^{27}$

\section{SURGICAL TREATMENT OF PSEUDOMEMBRANOUS COLITIS}

Surgery for $C$. difficile colitis is seldom indicated, being required in only $0.4 \%$ to $5 \%$ of cases. ${ }^{17}$ Surgery is reserved for complications of the disease such as fulminant colitis unresponsive to medical treatment, toxic megacolon, and free perforation. ${ }^{28-34}$ Approximately $3 \%$ to $20 \%$ of patients with PMC develop systemic signs of sepsis with fever, tachycardia, and leukocytosis. Aggressive medical management, preferably in an intensive care setting, should be instituted promptly.

Overall, $65 \%$ to $100 \%$ of patients with fulminant colitis eventually require surgery. Patients whose condition fails to improve within 48 hours of initiating treatment should be taken to surgery because delays in diagnosis and surgical intervention are direct predictors of mortality. Obviously, clinical deterioration in the presence of maximum medical management mandates urgent surgical intervention. Predicting the course of disease in individual patients is very difficult, and there are few, if any, markers to guide decision. A profound and rapid increase in leukocytosis $(30,000$ to $50,000)$ with bandemia sometimes immediately precedes sudden clinical deterioration with hypotension, hemodynamic instability, and shock. Patients who experience such a profound and rapid increase in leukocytosis should be operated urgently before clinical shock develops because the prognosis is dismal despite surgery once the need for preoperative vasopressors has arisen. 
In toxic patients, resolution of diarrhea may represent a symptom of deterioration rather than improvement. Diarrhea will stop because of severe colonic dysmotility with impending perforation. Urgent surgical intervention is indicated because of the high risk of perforation and the high mortality rate associated with this presentation. Once again, use of antidiarrheals (e.g., loperamide, Lomotil $\left.{ }^{\mathbb{R}}\right)$ is contraindicated in all cases of pseudomembranous enterocolitis as this may exacerbate toxin-mediated disease and precipitate colonic failure with toxic megacolon. Free perforation mandates surgical intervention.

Patients may present initially as a surgical emergency with typical toxic megacolon, symptoms and signs of systemic sepsis, and associated colonic dilatation. Urgent surgical intervention is indicated after initial resuscitation.

The overall mortality rate for surgical intervention varies from $25 \%$ to $70 \%$. These disappointing results underscore the aggressiveness of the disease. Increased mortality rates are seen in immunocompromised patients (particularly lung transplant patients), elderly patients, and patients with malignancies. Overall, the underlying medical condition, severity of the disease, and delays in diagnosis and surgical intervention directly affect the high mortality rates.

The operation of choice is subtotal colectomy with end ileostomy. Lesser procedures such as segmental colectomy, diverting stoma, nontherapeutic laparotomy, or cecostomy for vancomycin instillation are associated with a prohibitive mortality rate. The external appearance of the serosa does not reflect the severity of the mucosal disease and should not be used to gauge the resection margins should a lesser procedure be contemplated. Endoscopic findings are not predictive of the severity of the clinical disease, and endoscopic identification of pseudomembranes in a limited segment of the colon does not rule out pancolonic disease. Surgical removal of the entire colon removes the septic source and often results in an immediate and dramatic improvement in the patient's clinical condition while the patient is still on the operating room table. Primary anastomosis is contraindicated in these severely ill patients; anastomosis should be deferred 3 to 6 months after complete recovery.

\section{REFERENCES}

1. Rolfe RD, Helebian S, Finegold SM. Bacterial interference between Clostridium difficile and normal fecal flora. J Infect Dis 1981;143:470-475

2. Moyenuddin M, Williamson JC, Ohl CA. Clostridium difficile-associated diarrhea: current strategies for diagnosis and therapy. Curr Gastroenterol Rep 2002;4:279-286

3. Gerding DN, Johnson S, Peterson LR, et al. Clostridium difficile-associated diarrhea and colitis. Infect Control Hosp Epidemiol 1995;16:459-477
4. Warny M, Pepin J, Fang A, et al. Toxin production by an emerging strain of Clostridium difficile associated with outbreaks of severe disease in North America and Europe. Lancet 2005;366:1079-1084

5. Severe Clostridium difficile-associated disease in populations previously at low risk-four states, 2005. MMWR Morb Mortal Wkly Rep 2005;54:1201-1205

6. Hurley BW, Nguyen CC. The spectrum of pseudomembranous enterocolitis and antibiotic-associated diarrhea. Arch Intern Med 2002;162:2177-2184

7. Cleary RK. Clostridium difficile-associated diarrhea and colitis: clinical manifestations, diagnosis and treatment. Dis Colon Rectum 1998;41:1435-1449

8. Viscidi R, Wiley S, Bartlett JG. Isolation rates and toxigenic potential of Clostridium difficile isolates from various patient populations. Gastroenterology 1981;81:5-9

9. Oldfield EC III. Clostridium difficile-associated diarrhea: resurgence with a vengeance. Rev Gastroenterol Disord 2006; 6:79-96

10. Hecht JR, Olinger EJ. Clostridium difficile colitis secondary to intravenous vancomycin. Dig Dis Sci 1989;34:148149

11. Muto CA, Pokrywka M, Shutt K, et al. A large outbreak of Clostridium difficile-associated diarrhea with an unexpected proportion of deaths and colectomies at a teaching hospital following increased fluoroquinolone use. Infect Control Hosp Epidemiol 2005;26:273-280

12. Pepin J, Saheb N, Coulombe MA, et al. Emergence of fluoroquinolones as the predominant risk factor for Clostridium difficile-associated diarrhea: a cohort study during an epidemic in Quebec. Clin Infect Dis 2005;41: $1254-1260$

13. Khurana A, Vinayek N, Recco RA, et al. The incidence of Clostridium difficile-associated diarrhea after use of gatifloxacin and levofloxacin in an acute care facility. Clin Infect Dis 2004;39:602-603

14. Noren T. Outbreak from a high-toxin intruder: Clostridium difficile. Comment on: Lancet 2005;366:1079-1084. Lancet 2005;366:1053-1054

15. Pepin J, Valiquette L, Alary ME, et al. Clostridium difficileassociated diarrhea in a region of Quebec from 1991 to 2003: a changing pattern of disease severity. CMAJ 2004;171:466472

16. Vesoulis Z, Williams G, Matthews B. Pseudomembranous enteritis after proctocolectomy. Dis Colon Rectum 2000;43: $551-554$

17. Gonenne J, Pardi DS. Clostridium difficile: an update. Compr Ther 2004;30:134-140

18. Schroeder MS. Clostridium difficile-associated diarrhea. Am Fam Physician 2005;71:921-928

19. Bricker E, Garg R, Nelson R, et al. Antibiotic treatment for Clostridium difficile-associated diarrhea in adults. Cochrane Database Syst Rev 2005;25(1):CD004610

20. Alcantara CS, Guerrant RL. Update on Clostridium difficile infection. Curr Gastroenterol Rep 2000;2:310-314

21. Dendukuri N, Costa V, McGregor M, Brophy JM. Probiotic therapy for the prevention and treatment of Clostridium difficile-associated diarrhea: a systematic review. CMAJ 2005; 173:167-170

22. McFarland LV. Meta-analysis of probiotics for the prevention of antibiotic associated diarrhea and the treatment of Clostridium difficile disease. Am J Gastroenterol 2006;101: 812-822 
23. Bowden TA Jr, Mansberger AR Jr, Lykins LE. Pseudomembraneous enterocolitis: mechanism for restoring floral homeostasis. Am Surg 1981;47:178-183

24. Schwan A, Sjolin S, Trottestam U, et al. Relapsing Clostridium difficile enterocolitis cured by rectal infusion of normal faeces. Scand J Infect Dis 1984;16:211-215

25. Persky SE, Brandt LJ. Treatment of recurrent Clostridium difficile-associated diarrhea by administration of donated stool directly through the colonoscope. Am J Gastroenterol 2000;95: 3283-3285

26. Aas J, Gessert CE, Bakken JS. Recurrent Clostridium difficile colitis: case series involving 18 patients treated with donor stool administered via a nasogastric tube. Clin Infect Dis 2003;36: 580-585

27. Huebner ES, Surawicz CM. Treatment of recurrent Clostridium difficile diarrhea. Gastroenterol Hepatol 2006;2:203-208

28. Grundfest-Broniatowski S, Quader M, Alexander F, et al. Clostridium difficile colitis in the critically ill. Dis Colon Rectum 1996;39:619-623
29. Trudel JL, Deschenes M, Mayrand S, et al. Toxic megacolon complicating pseudomembranous enterocolitis. Dis Colon Rectum 1995;38:1033-1038

30. Dallal RM, Harbrecht BG, Boujoukas AJ, et al. Fulminant Clostridium difficile: an underappreciated and increasing cause of death and complications. Ann Surg 2002;235: 363-372

31. Jobe BA, Grasley A, Deveney KE, et al. Clostridium difficile colitis: an increasing hospital-acquired illness. Am J Surg 1995; 169:480-483

32. Lipsett PA, Samantaray DK, Tam ML, et al. Pseudomembranous colitis: a surgical disease? Surgery 1994;116:491-496

33. Medich DS, Lee KKW, Simmons RL, et al. Laparotomy for fulminant pseudomembranous colitis. Arch Surg 1992;127: 847-853

34. Morris JB, Zollinger RM, Stellato TA. The role of surgery in antibiotic-induced pseudomembranous enterocolitis. Am J Surg 1990;160:535-539 